\title{
CONNECTIONS ON MODULES OVER QUASI-HOMOGENEOUS PLANE CURVES
}

\author{
EIVIND ERIKSEN
}

\begin{abstract}
Let $k$ be an algebraically closed field of characteristic 0 , and let $A=k[x, y] /(f)$ be a quasi-homogeneous plane curve. We show that for any graded torsion free $A$-module $M$ without free summands, there exists a natural graded integrable connection, i.e. a graded $A$-linear homomorphism $\nabla: \operatorname{Der}_{k}(A) \rightarrow \operatorname{End}_{k}(M)$ that satisfy the derivation property and preserves the Lie product.

In particular, a torsion free module $N$ over the complete local ring $B=\widehat{A}$ admits a natural integrable connection if $A$ is a simple curve singularity, or if $A$ is irreducible and $N$ is a gradable module.
\end{abstract}

\section{INTRODUCTION}

Let $k$ be an algebraically closed field of characteristic 0 , let $A$ be a commutative $k$-algebra, and let $M$ be an $A$-module. We define a connection on $M$ to be an $A$-linear homomorphism

$$
\nabla: \operatorname{Der}_{k}(A) \rightarrow \operatorname{End}_{k}(M)
$$

that satisfy the derivation property, i.e. $\nabla_{D}(a m)=a \nabla_{D}(m)+D(a) m$ for all $D \in \operatorname{Der}_{k}(A), a \in A$ and $m \in M$. If $\nabla$ is also a homomorphism of Lie algebras, then we say that $\nabla$ is an integrable connection on $M$.

We say that $A$ is a quasi-homogeneous plane curve if $A=k[x, y] /(f)$ is a reduced, positively graded $k$-algebra given by a quasi-homogeneous polynomial $f \neq 0$. We shall denote its completion by $B=\widehat{A}$, and refer to $B$ as the complete local ring of a quasi-homogeneous plane curve singularity.

The simple curve singularities have been classified by Arnol'd [1] and Wall [6]. These singularities have complete local rings of the form

2000 Mathematics Subject Classification. Primary 13N10, 13N15.

Key words and phrases. Connections on modules, quasi-homogeneous plane curve singularities, simple curve singularities. 
$B=k[[x, y]] /(f)$, where $f$ is one of the following polynomials:

$$
\begin{array}{ll}
A_{n}: f=x^{2}+y^{n+1} & n \geq 1 \\
D_{n}: f=x^{2} y+y^{n-1} & n \geq 4 \\
E_{6}: f=x^{3}+y^{4} & \\
E_{7}: f=x^{3}+x y^{3} & \\
E_{8}: f=x^{3}+y^{5} &
\end{array}
$$

In particular, any simple curve singularity is of the form $B=\widehat{A}$ for a quasi-homogeneous plane curve $A=k[x, y] /(f)$. By abuse of language, we shall refer to the graded $k$-algebra $A$ as a simple curve singularity.

In this paper, we study existence of connections on an $A$-module $M$ when $A$ is a quasi-homogeneous plane curve and $M$ is a graded torsion free $A$-module. A connection $\nabla: \operatorname{Der}_{k}(A) \rightarrow \operatorname{End}_{k}(M)$ is graded if $\nabla_{D}\left(M_{w}\right) \subseteq M_{w+\lambda}$ for any homogeneous derivation $D \in \operatorname{Der}_{k}(A)$ of weight $\lambda$ and any integer $w \in \mathbf{Z}$. We show the following result:

Theorem 1. Let $A=k[x, y] /(f)$ be a quasi-homogeneous plane curve, and let $M$ be a graded torsion free A-module. If $A$ is irreducible or a simple curve singularity and $M$ is without free summands, then there is a natural graded integrable connection on $M$.

In fact, we believe that any graded torsion free module without free summands over an arbitrary quasi-homogeneous plane curve admits a natural graded integrable connection. We give a sufficient condition in lemma 6, and show that it is satisfied if $A=k[x, y] /\left(y\left(x^{n}-y^{m}\right)\right)$ for positive integers $m, n \in \mathbf{N}$ with $(m, n)=1$ and if $M$ has rank one. However, we have not been able to find a proof in the general case.

We say that a torsion free $B$-module $N$ is gradable if it is of the form $N \cong \widehat{M}$ for some graded torsion free $A$-module $M$. If $B$ is the complete local ring of a simple curve singularities, then it follows from theorem (15.14) in Yoshino [7] that any torsion free $B$-module is gradable.

Corollary 2. Any torsion free module over the complete local ring of a simple curve singularity admits a natural integrable connection.

Corollary 3. Any gradable torsion free module over the complete local ring of an irreducible quasi-homogeneous plane curve singularity admits a natural integrable connection. 


\section{Quasi-Homogeneous Plane CURVES}

Let $k$ be an algebraically closed field. We define a positively graded $k$-algebra to be a finitely generated $\mathbf{N}_{0}$-graded $k$-algebra

$$
A=\underset{w \in \mathrm{N}_{0}}{\oplus} A_{w}
$$

with $A_{0}=k$. In this paper, we shall assume that $A$ is a positively graded, reduced $k$-algebra of the form $A=k[x, y] /(f)$, where $f \neq 0$. This implies that the complete local ring $\widehat{A}$ is a quasi-homogeneous plane curve singularity, and we shall refer to the graded ring $A$ as a quasi-homogeneous plane curve.

The simple curve singularities have been classified by Arnol'd [1] and Wall [6]. These singularities have complete local rings of the form $B=k[[x, y]] /(f)$, where $f$ is one of the following polynomials:

$$
\begin{array}{rlrl}
A_{n} & : f=x^{2}+y^{n+1} & n \geq 1 \\
D_{n}: f=x^{2} y+y^{n-1} & n \geq 4 \\
E_{6}: f=x^{3}+y^{4} & & \\
E_{7}: f=x^{3}+x y^{3} & & \\
E_{8}: f=x^{3}+y^{5} & &
\end{array}
$$

In particular, the complete local ring of any simple curve singularity is of the form $B=k[[x, y]] /(f) \cong \widehat{A}$, where $A=k[x, y] /(f)$ is a quasihomogeneous plane curve. By abuse of language, we shall refer to the graded $k$-algebra $A$ as a simple curve singularity.

We introduce some notation that will be used throughout this paper: Let $k[x, y]$ be a positively graded polynomial $k$-algebra, with grading given by positive weights $w_{x}=\operatorname{deg}(x), w_{y}=\operatorname{deg}(y) \in \mathbf{N}$ satisfying $\left(w_{x}, w_{y}\right)=1$, and let $f$ be a homogeneous element in $k[x, y]$ of positive weight $w_{f}=\operatorname{deg}(f) \in \mathbf{N}$. We recall that the homogeneous elements $f \in k[x, y]$ of weight $w_{f}$ are the quasi-homogeneous polynomials of weight $w_{f}$, i.e. the polynomials $f \in k[x, y]$ that satisfy

$$
f\left(\alpha^{w_{x}} \cdot x, \alpha^{w_{y}} \cdot y\right)=\alpha^{w_{f}} \cdot f(x, y)
$$

for all $\alpha \in k^{*}$. We consider $A=k[x, y] /(f)$ with the induced grading, which is a positively graded $k$-algebra.

Lemma 1. We have that $f=u \cdot f_{1} \cdots f_{r}$, where $u \in k^{*}$ is a unit in $k[x, y]$, and $f_{i} \in k[x, y]$ is an irreducible polynomial given by $f_{i}=x$, $f_{i}=y$ or $f_{i}=x^{w_{y}}+a_{i} y^{w_{x}}$ for some $a_{i} \in k^{*}$ for $1 \leq i \leq r$.

Proof. In case $w_{x}=w_{y}=1$, it is enough to show that any quasihomogeneous polynomial has a factorization into linear polynomials. 
This is an elementary fact, since $k$ is algebraically closed. In the general case, any irreducible quasi-homogeneous polynomial with more than one summand must include $x^{s w_{y}}$ and $y^{s w_{x}}$. By an argument similar to the one in case $w_{x}=w_{y}=1$, irreducibility implies that $s=1$.

For $1 \leq i \leq r$, we denote the weight of $f_{i}$ by $w_{i}=\operatorname{deg}\left(f_{i}\right) \in \mathbf{N}$, and write $A_{i}=A /\left(f_{i}\right)$ for the corresponding positively graded domain. We assume that $f_{i} \neq f_{j}$ when $i \neq j$, which means that $A$ is reduced.

For any graded $k$-algebra $R$, let $Q(R)$ be the graded total ring of fractions of $R$, i.e. the localization $Q(R)=S^{-1} R$, where $S$ is the set a homogeneous non-zero divisors in $R$. Then the natural map $R \rightarrow Q(R)$ is an injective graded homomorphism, and we identify $R$ with its image in $Q(R)$. We define the graded normalization $\widetilde{R}$ of $R$ to be the integral closure of $R$ in $Q(R)$, and write $n: R \rightarrow \widetilde{R}$ for the graded normalization map.

It follows from lemma 1 that there is an isomorphism $\widetilde{A}_{i} \cong k\left[t_{i}\right]$ of graded $k$-algebras for $1 \leq i \leq r$, with $d_{i}=\operatorname{deg}\left(t_{i}\right) \in \mathbf{N}$ given by

$$
d_{i}= \begin{cases}w_{y} & \text { if } f_{i}=x \\ w_{x} & \text { if } f_{i}=y \\ 1 & \text { if } f_{i}=x^{w_{y}}+a_{i} y^{w_{x}}\end{cases}
$$

Moreover, the graded normalization map $n_{i}: A_{i} \rightarrow k\left[t_{i}\right]$ is the graded algebra homomorphism given by

$$
x \mapsto\left\{\begin{array} { l l } 
{ 0 } & { \text { if } f _ { i } = x } \\
{ t _ { i } } & { \text { if } f _ { i } = y } \\
{ t _ { i } ^ { w _ { x } } } & { \text { if } f _ { i } = x ^ { w _ { y } } + a _ { i } y ^ { w _ { x } } }
\end{array} \quad y \mapsto \left\{\begin{array}{ll}
t_{i} & \text { if } f_{i}=x \\
0 & \text { if } f_{i}=y \\
b_{i} t_{i}^{w_{y}} & \text { if } f_{i}=x^{w_{y}}+a_{i} y^{w_{x}}
\end{array}\right.\right.
$$

for $1 \leq i \leq r$, where $b_{i} \in k^{*}$ is a solution of $a_{i} b_{i}^{w_{x}}=-1$. Since we have that

$$
\widetilde{A} \cong \underset{1 \leq i \leq r}{\oplus} \widetilde{A}_{i}
$$

there is an isomorphism $\widetilde{A} \cong k\left[t_{1}\right] \oplus \ldots \oplus k\left[t_{r}\right]$ of graded $k$-algebras. We recall that the graded $k$-algebra $k\left[t_{1}\right] \oplus \ldots \oplus k\left[t_{r}\right]$ has $k$-linear base $\left\{t_{i}^{l_{i}}=\left(0, \ldots, t_{i}^{l_{i}}, \ldots, 0\right): 1 \leq i \leq r, l_{i} \in \mathbf{N}_{0}\right\}$, multiplication given by $t_{i}^{l_{i}} \cdot t_{j}^{m_{j}}=\delta_{i j} \cdot t_{i}^{l_{i}+m_{j}}$ for $1 \leq i, j \leq r, l_{i}, m_{j} \in \mathbf{N}_{0}$, and $k$-algebra structure given by the diagonal map $k \rightarrow k^{r}$. The grading of $k\left[t_{1}\right] \oplus \ldots \oplus k\left[t_{r}\right]$ is given by $\operatorname{deg}\left(t_{i}^{l_{i}}\right)=l_{i} d_{i}$ for $1 \leq i \leq r, l_{i} \in \mathbf{N}_{0}$, hence it is a positively graded $k^{r}$-algebra. We may identify $A$ with a graded subring of $k\left[t_{1}\right] \oplus \ldots \oplus k\left[t_{r}\right]$ via the graded normalization map $n: A \rightarrow \widetilde{A}$. 


\section{Derivations}

Let $k$ be an algebraically closed field of characteristic 0 , and let $R$ be any graded $k$-algebra. We recall that the derivation module $\operatorname{Der}_{k}(R)$ is the left $R$-module consisting of all $k$-linear operators $Q: R \rightarrow R$ satisfying the Leibniz rule

$$
Q(r s)=Q(r) s+r Q(s) \text { for all } r, s \in R .
$$

We say that a derivation $Q \in \operatorname{Der}_{k}(R)$ is homogeneous of weight $\lambda \in \mathbf{Z}$ if $Q\left(A_{w}\right) \subseteq A_{w+\lambda}$ for all $w \in \mathbf{N}$.

Let $A=k[x, y] /(f)$ be a quasi-homogeneous plane curve. It is wellknown that $\operatorname{Der}_{k}(A)$ is generated, as a left $A$-module, by the Euler derivation $E$ and the Koszul derivation $D$, given by

$$
\begin{aligned}
& E=w_{x} x \cdot \partial_{x}+w_{y} y \cdot \partial_{y} \\
& D=f_{y} \cdot \partial_{x}-f_{x} \cdot \partial_{y}
\end{aligned}
$$

We write $\partial_{x}=\partial / \partial x$ and $\partial_{y}=\partial / \partial y$ for the partial derivations, and $f_{x}=\partial_{x}(f)$ and $f_{y}=\partial_{y}(f)$ for the partial derivatives of $f$.

We claim that any derivation $P: A \rightarrow A$ has a canonical extension to a derivation $\widetilde{P}: \widetilde{A} \rightarrow \widetilde{A}$. If $r=1$, the claim follows from the graded version of a theorem by Seidenberg [5]. If $r>1$, any derivation $P: A \rightarrow A$ induces a derivation $P_{i}: A_{i} \rightarrow A_{i}$ for $1 \leq i \leq r$, since $E\left(f_{i}\right)=w_{i} \cdot f_{i}$ and $D\left(f_{i}\right)=f_{i} \cdot\left[\partial_{x}\left(f_{i}\right) \cdot \partial_{y}\left(g_{i}\right)-\partial_{y}\left(f_{i}\right) \cdot \partial_{x}\left(g_{i}\right)\right]$ with $g_{i}=f / f_{i}$, and $\widetilde{P}=\left(\widetilde{P}_{1}, \ldots, \widetilde{P}_{r}\right): \widetilde{A} \rightarrow \widetilde{A}$ is an extension of $P: A \rightarrow A$.

Lemma 2. We have that $\widetilde{E}=d_{1} t_{1} \cdot \partial_{1}+\cdots+d_{r} t_{r} \cdot \partial_{r} \in \operatorname{Der}_{k}(\widetilde{A})$, where $\partial_{i}=\partial / \partial t_{i}$ for $1 \leq i \leq r$.

Proof. The Euler derivation $E: A \rightarrow A$ is characterized by $E(h)=w \cdot h$ for any homogeneous element $h \in A$ of weight $w$. Since $n: A \rightarrow \widetilde{A}$ is a graded algebra homomorphism, it follows that $\widetilde{E}: \widetilde{A} \rightarrow \widetilde{A}$ can be characterized in the same way, and $\widetilde{E}=d_{1} t_{1} \cdot \partial_{1}+\cdots+d_{r} t_{r} \cdot \partial_{r}$.

Let $\Gamma_{i} \subseteq \mathbf{N}_{0}$ be the semigroup given by $\Gamma_{i}=\left\{\underset{\sim}{\gamma} \in \mathbf{N}_{0}: t_{i}^{\gamma} \in A\right\}$ for $1 \leq i \leq r$, where we identify $A$ with its image in $\widetilde{A}$. Hence we have

$$
\gamma \in \Gamma_{i} \Longleftrightarrow\left(0, \ldots, t_{i}^{\gamma}, \ldots, 0\right) \in A
$$

for any $\gamma \in \mathbf{N}_{0}$. We remark that if $r>1$, then $0 \notin \Gamma_{i}$ for $1 \leq i \leq r$, so $\Gamma_{i}$ should be thought of as a semigroup without zero in that case. It is clear that $\mathbf{N}_{0} \backslash \Gamma_{i}$ is finite for $1 \leq i \leq r$, hence we may define $c_{i}=\min \left\{\gamma \in \Gamma_{i}: \gamma+\mathbf{N}_{0} \subseteq \Gamma_{i}\right\}$ to be the conductor of $\Gamma_{i}$, and $g_{i}=c_{i}-1$ to be the Frobenius number of $\Gamma_{i}$. 
Proposition 3. We have that $\widetilde{D}=\beta_{1} t_{1}^{c_{1}} \cdot \partial_{1}+\cdots+\beta_{r} t_{r}^{c_{r}} \cdot \partial_{r} \in \operatorname{Der}_{k}(\widetilde{A})$ for some constants $\beta_{1}, \ldots, \beta_{r} \in k^{*}$, where $\partial_{i}=\partial / \partial t_{i}$ for $1 \leq i \leq r$. Moreover, the conductor $c_{i}$ of $\Gamma_{i}$ is given by

$$
c_{i}=\frac{w_{f}-w_{i}}{d_{i}}+c\left(A_{i}\right)
$$

for $1 \leq i \leq r$, where $c\left(A_{i}\right)$ is the conductor of the irreducible quasihomogeneous curve $A_{i}$.

Proof. If $r=1$, then it is well-known that $\widetilde{D}=\beta_{1} t_{1}^{c_{1}} \cdot \partial_{1}$ for some $\beta_{1} \in k^{*}$, see for instance section 6 of Eriksen [3]. Hence we may assume that $r>1$. Let $g_{i}=f / f_{i} \in k[x, y]$ for $1 \leq i \leq r$. It is clear that $n_{I}\left(g_{i}\right)=0$ if $I \neq i$ and that $n_{i}\left(g_{i}\right) \neq 0$. In fact, we see that $n_{i}\left(g_{i}\right) \in k\left[t_{i}\right]$ is homogeneous of degree $w-w_{i}$, and therefore of the form

$$
n_{i}\left(g_{i}\right)=\beta_{i}^{\prime} \cdot t_{i}^{\frac{w_{f}-w_{i}}{d_{i}}}
$$

for some $\beta_{i}^{\prime} \in k^{*}$, hence $\frac{w-w_{i}}{d_{i}} \in \Gamma_{i}$. For $1 \leq i \leq r$, we also see that any element $h_{i} \in k[x, y]$ with the property that $n_{I}\left(h_{i}\right)=0$ for $I \neq i$ must be of the form $h_{i}=q \cdot g_{i}$ with $q \in k[x, y]$. Therefore, $c_{i}=\frac{w-w_{i}}{d_{i}}+c\left(A_{i}\right)$, where $c\left(A_{i}\right)$ is the conductor of $A_{i}$. Explicitly, $c\left(A_{i}\right)=0$ if $f_{i}=x$ or $f_{i}=y$, and $c\left(A_{i}\right)=\left(w_{x}-1\right)\left(w_{y}-1\right)$ otherwise.

On the other hand, we see that the derivation $D_{i} \in \operatorname{Der}_{k}\left(A_{i}\right)$ induced by the Koszul derivation $D \in \operatorname{Der}_{k}(A)$ has the form

$$
D_{i}=g_{i} \cdot\left(\partial_{y}\left(f_{i}\right) \partial_{x}-\partial_{x}\left(f_{i}\right) \partial_{y}\right)
$$

for $1 \leq i \leq r$. Since $\partial_{y}\left(f_{i}\right) \partial_{x}-\partial_{x}\left(f_{i}\right) \partial_{y}$ is the Koszul derivation on $A_{i}$, its natural extension to $\widetilde{A}_{i}$ has the form $\beta_{i}^{\prime \prime} t_{i}^{c\left(A_{i}\right)} \cdot \partial_{i}$ for some constant $\beta_{i}^{\prime \prime} \in k^{*}$, see for instance section 6 of Eriksen [3]. It follows that $\widetilde{D}_{i} \in \operatorname{Der}_{k}\left(\widetilde{A}_{i}\right)$ has the form

$$
\widetilde{D}_{i}=n_{i}\left(g_{i}\right) \cdot \beta_{i}^{\prime \prime} t_{i}^{c\left(A_{i}\right)} \cdot \partial_{i}=\beta_{i} t^{c_{i}} \cdot \partial_{i}
$$

with $\beta_{i}=\beta_{i}^{\prime} \cdot \beta_{i}^{\prime \prime} \in k^{*}$.

We remark that $\widetilde{D}=q \cdot \widetilde{E}$ considered as elements in $\operatorname{Der}_{k}(\widetilde{A})$, where $q \in \widetilde{A}$ is given by $q=\left(\frac{\beta_{1}}{d_{1}} t_{1}^{g_{1}}, \ldots, \frac{\beta_{r}}{d_{r}} t_{r}^{g_{r}}\right)$. In fact, $q \in(A: m)$, where $m=(x, y) \subseteq A$ denotes the maximal homogeneous ideal of $A$, and one may show that $\operatorname{Der}_{k}(A)=(A: m) \cdot E$.

\section{Graded torsion free modules}

Let $k$ be an algebraically closed field, let $A=k[x, y] /(f)$ be a quasihomogeneous plane curve, and let $M$ be a finitely generated, graded $A$-module. If the natural map $M \rightarrow M \otimes_{A} Q(A)$ is injective, we say 
that $M$ is torsion free. Notice that $M$ is torsion free if and only if it is maximal Cohen-Macaulay, i.e. depth $M_{m}=1$, where $m=(x, y) \subseteq A$ is the maximal homogeneous ideal of $A$.

Lemma 4. If $M$ is a torsion free $A$-module, then we may identify $M$ with a graded submodule of

$$
\underset{1 \leq i \leq r}{\oplus} \widetilde{A}_{i}^{s_{i}} \cong k\left[t_{1}\right]^{s_{1}} \oplus \cdots \oplus k\left[t_{r}\right]^{s_{r}}
$$

where $s_{i} \geq 0$ is the rank of $M \otimes_{A} A_{i}$ as an $A_{i}$-module for $1 \leq i \leq r$.

Proof. It is clear that $M \otimes_{A} A_{i}$ has a rank $s_{i} \geq 0$ as an $A_{i}$-module for $1 \leq i \leq r$, since $Q\left(A_{i}\right) \cong k\left[t_{i}, t_{i}^{-1}\right]$. We have

$$
M \otimes_{A} Q(A) \cong \underset{1 \leq i \leq r}{\oplus} M \otimes_{A} Q\left(A_{i}\right)
$$

and $M \otimes_{A} Q\left(A_{i}\right) \cong\left(M \otimes_{A} A_{i}\right) \otimes_{A_{i}} Q\left(A_{i}\right) \cong Q\left(A_{i}\right)^{s_{i}}$ for $1 \leq i \leq r$. Hence the natural map $M \hookrightarrow M \otimes_{A} Q(A) \cong \oplus_{i} Q\left(A_{i}\right)^{s_{i}}$ identifies $M$ with a graded submodule of $\oplus_{i} Q\left(A_{i}\right)^{s_{i}}$. Since $M$ is finitely generated, we may identify $M$ with a graded submodule of $\oplus_{i} \widetilde{A}_{i} s_{i}=\oplus_{i} k\left[t_{i}\right]^{s_{i}}$ by multiplying with a suitable denominator.

For $1 \leq i \leq r$, we choose a set $\left\{e_{i j}: 1 \leq j \leq s_{i}\right\}$ of homogeneous generators for the graded $\widetilde{A}_{i}$-module $\widetilde{A}_{i}{ }^{s_{i}}$, and write $f_{i j}=\operatorname{deg}\left(e_{i j}\right) \in \mathbf{Z}$ for their weights. We consider $\left\{e_{i j}: 1 \leq i \leq r, 1 \leq j \leq s_{i}\right\}$ as a set of homogeneous generators for the graded $\widetilde{A}$-module $\oplus_{i} \widetilde{A}_{i} s_{i}$, where $\widetilde{A}$ acts on $\oplus_{i} \widetilde{A}_{i} s_{i}$ via the projections $\pi_{i}: \widetilde{A} \rightarrow \widetilde{A}_{i}$ for $1 \leq i \leq r$.

Let $M \subseteq \oplus_{i} \widetilde{A}_{i} s_{i}$ be a graded submodule, let $\left\{m_{l}: 1 \leq l \leq L\right\}$ be a set of homogeneous generators of $M$, and write $w_{l}=\operatorname{deg}\left(m_{l}\right) \in \mathbf{Z}$ for their weights. Explicitly, $m_{l}$ is of the form

$$
m_{l}=\sum_{i, j} a_{i j}(l) \cdot e_{i j}
$$

for $1 \leq l \leq L$, where $a_{i j}(l) \in \widetilde{A}_{i}$ is homogeneous of weight $w_{l}-f_{i j}$ for $1 \leq i \leq r, 1 \leq j \leq s_{i}$. Let us write $\pi_{i}: \oplus_{i} \widetilde{A}_{i}{ }^{s_{i}} \rightarrow \widetilde{A}_{i}{ }^{s_{i}}$ for the $i$ 'th projection. We may assume that the generating set $\left\{m_{l}: 1 \leq l \leq L\right\}$ satisfy the following condition:

(C1) For all $1 \leq i \leq r, 1 \leq j \leq s_{i}$, there is a homogeneous generator $m_{l}$ with $\pi_{i}\left(m_{l}\right)=e_{i j}$

In particular, condition (C1) implies that we may choose set of homogeneous generators containing $\left\{e_{1}, \ldots, e_{s}\right\}$ when $r=1$. 


\section{Connections on GRAded MOdules}

Let $k$ be an algebraically closed field of characteristic 0 , and let $R$ be any graded $k$-algebra. We define a connection on a graded $R$-module $V$ to be an $R$-linear homomorphism

$$
\nabla: \operatorname{Der}_{k}(R) \rightarrow \operatorname{End}_{k}(V)
$$

that satisfies the derivation property, i.e. $\nabla_{D}(r v)=r \nabla_{D}(v)+D(r) v$ for all $D \in \operatorname{Der}_{k}(R), r \in R, v \in V$. We say that $\nabla$ is graded, or homogeneous of weight 0 , if $\nabla_{D}\left(V_{w}\right) \subseteq V_{w+\lambda}$ for any homogeneous derivation $D \in \operatorname{Der}_{k}(R)$ of weight $\lambda$ and for any integer $w \in \mathbf{Z}$, and that $\nabla$ is integrable if it is a Lie algebra homomorphism.

Let $A=k[x, y] /(f)$ be a quasi-homogeneous plane curve, and let $M$ be a finitely generated, graded torsion free $A$-module. We may assume that $M \subseteq \oplus_{i} \widetilde{A}_{i}{ }^{s_{i}}$ is a graded submodule that satisfies (C1).

The Euler derivation $E: A \rightarrow A$ has a canonical extension to a derivation $\widetilde{E}=\left(\widetilde{E}_{1}, \ldots, \widetilde{E}_{r}\right): \widetilde{A} \rightarrow \widetilde{A}$, and an induced $k$-linear action $\nabla_{E} \in \operatorname{End}_{k}\left(\oplus_{i} \widetilde{A}_{i} s_{i}\right)$ that satisfies the derivation property. The induced action $\nabla_{E}$ is characterized by $\nabla_{E}(h)=w \cdot h$ for any homogeneous element $h \in \oplus_{i} \widetilde{A}_{i} s_{i}$ of weight $w$.

The Koszul derivation $D: A \rightarrow A$ has a canonical extension to a derivation $\widetilde{D}=\left(\widetilde{D}_{1}, \ldots, \widetilde{D}_{r}\right): \widetilde{A} \rightarrow \widetilde{A}$, and $\widetilde{D}=q \cdot \widetilde{E}$ in $\operatorname{Der}_{k}(\widetilde{A})$ with $q \in(A: m)$. Hence there is a unique extension of the $k$-linear action $\nabla_{E}$ to a connection $\nabla: \operatorname{Der}_{k}(A) \rightarrow \operatorname{End}_{k}(M)$, with induced $k$-linear action $\nabla_{D} \in \operatorname{End}_{k}\left(\oplus_{i} \widetilde{A}_{i}{ }^{s_{i}}\right)$ given by $\nabla_{D}(h)=q \cdot \nabla_{E}(h)=q w \cdot h$ for any homogeneous element $h \in \oplus_{i} \widetilde{A}_{i}{ }^{s_{i}}$ of weight $w$. We remark that $\nabla$ is a graded integrable connection.

Lemma 5. Let $M \subseteq \oplus_{i} \widetilde{A}_{i}{ }^{s_{i}}$ be a graded submodule. If $\nabla_{D}(M) \subseteq M$, then $\nabla$ induces a graded integrable connection on $M$.

For a graded submodule $M \subseteq \oplus_{i} \widetilde{A}_{i}{ }^{s_{i}}$ that satisfies (C1), we consider the following conditions:

(C2) For $1 \leq i \leq r, 1 \leq j \leq s_{i}$, we have $t_{i}^{g_{i}} e_{i j} \in M$

(C3) For $1 \leq i \leq r, 1 \leq j \leq s_{i}$, we have $f_{i j}=\lambda$ for some $\lambda \in \mathbf{Z}$.

We remark that given a description of $M \subseteq \oplus_{i} \widetilde{A}_{i} s_{i}$ in concrete terms, it is easy to check if the conditions (C2) and (C3) hold.

Lemma 6. Let $M \subseteq \bigoplus_{i} \widetilde{A}_{i} s_{i}$ be a graded submodule. If $M$ satisfies (C1) and (C2), then $\nabla_{D}(M) \subseteq M$.

Proof. If $\gamma \in \Gamma_{i}$, then $t_{i}^{\gamma} \in A$ by definition, hence condition (C1) implies that $t_{i}^{\gamma} e_{i j} \in M$. In particular, $t_{i}^{\gamma} e_{i j} \in M$ for all $\gamma \geq g_{i}$ by $(\mathrm{C} 1)$ and 
(C2). On the other hand, the homogeneous generator $m_{l}$ of $M$ of weight $w_{l}$ has the form $m_{l}=\sum_{i j} a_{i j}(l) \cdot e_{i j}$ for $1 \leq l \leq L$, where $a_{i j}(l) \in \widetilde{A}_{i}$ is homogeneous of weight $w_{l}-f_{i j} \geq 0$ for $1 \leq i \leq r, 1 \leq j \leq s_{i}$. Hence we have that $\nabla_{D}\left(m_{l}\right)=w_{l} q \cdot m_{l} \in M$ for $1 \leq l \leq L$, and it follows that $\nabla_{D}(M) \subseteq M$.

Lemma 7. Let $M \subseteq \oplus_{i} \widetilde{A}_{i}{ }^{s}$ be a graded submodule. If $M$ satisfies (C1) and (C3), then $\nabla_{D}(M(-\lambda)) \subseteq M(-\lambda)$.

Proof. We may assume that $\lambda=0$, hence $w_{l}=\operatorname{deg}\left(m_{l}\right) \geq 0$ for all $l$. If $w_{l}=0$, then $\operatorname{deg}\left(a_{i j}(l)\right)=w_{l}-f_{i j}=0$ for all $i, j$, hence $\nabla_{D}\left(m_{l}\right)=0$. If $w_{l}>0$, then $\operatorname{deg}\left(a_{i j}(l)\right)=w_{l}-f_{i j}=w_{l}>0$ for all $i, j$, hence $q a_{i j}(l) \in A$ and $\nabla_{D}\left(m_{l}\right)=\sum_{i j} w_{l} q a_{i j}(l) \cdot e_{i j} \in M$.

In particular, if $M$ is free and indecomposable, then we may identify $M$ with the submodule of $\widetilde{A}=\oplus_{i} \widetilde{A}_{i}$ generated by $e_{11}+e_{21}+\cdots+e_{r 1}$. Hence (C3) holds with $\lambda=f_{11}=\cdots=f_{r 1}$, and there is a natural integrable graded connection on $M(-\lambda)$ by lemma 5 and lemma 7 .

Proposition 8. If $A$ is irreducible and $M$ is indecomposable and not free, then $M$ satisfies (C1) and (C2).

Proof. Let $M \subseteq \widetilde{A}^{s}$ be a graded submodule that satisfies (C1). We may assume that the set $\left\{m_{l}: 1 \leq l \leq L\right\}$ of homogeneous generators of $M$ satisfies $L>s$ and $m_{l}=e_{l}$ for $1 \leq l \leq s$. For $l>s, m_{l} \in M$ has the form

$$
m_{l}=\sum_{1 \leq j \leq s} a_{j}(l) \cdot e_{j}
$$

where $a_{j}(l) \in \widetilde{A}$ is homogeneous of weight $\operatorname{deg}\left(a_{j}(l)\right)=w_{l}-f_{j}$ for $1 \leq j \leq s$. We may assume that $a_{j}(l)=0$ when $w_{l}-f_{j} \in \Gamma$, since $e_{j} \in M$. We may also assume that $a_{j}(l)=0$ or $a_{J}(l)=0$ when $j \neq J$ and $\operatorname{deg}\left(a_{J}(l)\right)-\operatorname{deg}\left(a_{j}(l)\right) \in \Gamma$, since $M$ is indecomposable.

We claim that these assumptions imply that $t^{g} e_{j} \in M$ for $1 \leq j \leq s$. In fact, $a_{j}(l) \neq 0$ for some integer $l>s$, otherwise $A \cdot e_{j}$ would be a direct summand of $M$. By assumption, $\operatorname{deg}\left(a_{j}(l)\right) \notin \Gamma$, and since $\Gamma$ is symmetric, it follows that $g-\operatorname{deg}\left(a_{j}(l)\right) \in \Gamma$ and therefore that $b=t^{g-\operatorname{deg}\left(a_{j}(l)\right)} \in A$. Hence $b \cdot m_{l} \in M$ can be expressed as

$$
b \cdot m_{l}=c t^{g} \cdot e_{j}+\sum_{J \neq j} b a_{J}(l) \cdot e_{J}
$$

for some $c \in k^{*}$. If $a_{J}(l) \neq 0$ for some $J \neq j$, then by assumption, $\operatorname{deg}\left(a_{j}(l)\right)-\operatorname{deg}\left(a_{J}(l)\right) \notin \Gamma$, and $g-\left(\operatorname{deg}\left(a_{j}(l)\right)-\operatorname{deg}\left(a_{J}(l)\right)\right) \in \Gamma$. It follows that $\operatorname{deg}\left(b a_{J}(l)\right) \in \Gamma$ and that $b a_{J}(l) \in A$ when $J \neq j$ and 
$a_{J}(l) \neq 0$. Hence $b a_{J}(l) \cdot e_{J} \in M$ for all $J \neq j$, and this implies that $t^{g} e_{j} \in M$.

It seems probable that any indecomposable module $M$ that is not free satisfies (C1) and (C2), even when $A$ has more than one irreducible component, but we have not been able to find a proof in the general case. To illustrate the combinatorial problems involved, we an example. This example also shows that there are modules of rank one that does not satisfy (C3).

Let $A=k[x, y] /\left(y\left(x^{n}-y^{m}\right)\right)$ for some natural numbers $m, n \in \mathbf{N}$ with $(m, n)=1$. Then $A \cong k\left[\left(t_{1}, t_{2}^{m}\right),\left(0, t_{2}^{n}\right)\right] \subseteq k\left[t_{1}\right] \oplus k\left[t_{2}\right]$ and the semigroups $\Gamma_{i}$ are given by

$$
\begin{aligned}
& \Gamma_{1}=\{n, n+1, n+2, \ldots\} \\
& \Gamma_{2}=\langle n, n+m, n+2 m, \ldots, n+(n-1) m\rangle
\end{aligned}
$$

with $g_{1}=n-1$ and $g_{2}=m n-m=m(n-1)$. Let $M$ be a finitely generated graded torsion free $A$-module of rank one, and assume that $M$ is not free. Then we may assume that $M$ has a set of homogeneous generators containing one of the following sets:

(1) $\left\{e_{11}+e_{21}, t_{2}^{h} \cdot e_{21}\right\}$ for $h \in \mathbf{N} \backslash \Gamma_{2}$

(2) $\left\{e_{11}+t_{2}^{h} \cdot e_{21}, e_{21}\right\}$ for $h \in \mathbf{N} \backslash\langle m, n\rangle$

In the first case, we see that $g_{2}-h \in\langle m, n\rangle$, hence there is an element $a=\left(*, t_{2}^{g_{2}-h}\right) \in A$ and $a \cdot t_{2}^{h} e_{21}=t_{2}^{g_{2}} e_{21} \in M$. In fact, $h-n \notin\langle m, n\rangle$ since $h \notin \Gamma_{2}$, hence $g\left(A_{2}\right)-(h-n)=g_{2}-h \in\langle m, n\rangle$. Moreover, $\left(t_{1}, t_{2}^{m}\right)^{n-1} \cdot\left(e_{11}+e_{21}\right)=t_{1}^{g_{1}} e_{11}+t_{2}^{g_{2}} e_{21} \in M$. It follows that condition (C2) holds. In the second case, $\left(t_{1}, t_{2}^{m}\right)^{n-1} \cdot e_{21}=t_{2}^{g_{2}} e_{21} \in M$, and $\left(t_{1}, t_{2}^{m}\right)^{n-1} \cdot\left(e_{11}+t^{h} e_{21}\right)=t_{1}^{g_{1}} e_{11}+t_{2}^{g_{2}+h} e_{21} \in M$. Since $g_{2}+h \in \Gamma_{2}$, it follows that condition $(\mathrm{C} 2)$ holds. We conclude that $(\mathrm{C} 2)$ holds in both cases, while (C3) holds only in the first case.

Theorem 9. Let $A=k[x, y] /(f)$ be a quasi-homogeneous plane curve, and let $M$ be a finitely generated graded torsion free A-module. If $A$ is irreducible or a simple curve singularity and $M$ is without free summands, then there is a natural graded integrable connection on $M$.

Proof. By lemma 5 and 6, it is enough to show that there is a graded embedding $M \subseteq \oplus_{i} \widetilde{A}_{i}{ }^{s_{i}}$ such that conditions (C1) - (C2) hold when $M$ is indecomposable. Hence the theorem follows from proposition 8 when $A$ is irreducible. When $A$ is a simple curve singularity with more than one irreducible component, it is straight-forward to verify that (C2) holds in each case, using the complete list of indecomposable modules given in the appendix of Greuel and Knörrer [4]. 


\section{Connections on modules over CURve Singularities}

Let $k$ be an algebraically closed field of characteristic 0 , and let $A=k[x, y] /(f)$ be a quasi-homogeneous plane curve. We denote its $m$-adic completion by $B=\widehat{A} \cong k[[x, y]] /(f)$, where $m=(x, y) \subseteq A$ is the maximal homogeneous ideal of $A$. Hence $B$ is the complete local ring of a quasi-homogeneous plane curve singularity.

If $M$ is a graded torsion free $A$-module, then $\widehat{M}$ is a torsion free $B$-module. We say that a torsion free $B$-module $N$ is gradable if there is a graded torsion free $A$-module $M$ such that $\widehat{M} \cong N$. We remark that by theorem (15.14) in Yoshino [7] and the fact that the simple curve singularities have finite CM representation type, it follows that any torsion free module over a simple curve singularity is gradable.

Corollary 10. Any torsion free module over the complete local ring of a simple curve singularity admits a natural integrable connection.

Corollary 11. Any gradable torsion free module over the complete local ring of an irreducible quasi-homogeneous plane curve singularity admits a natural integrable connection.

\section{ACKNOWLEDGEMENTS}

The results concerning simple curve singularities were obtained while I worked on my Master's thesis Eriksen [2] at the University of Oslo. I wish to thank my thesis supervisor, professor O.A. Laudal, for introducing me to this problem and for sharing his ideas.

\section{REFERENCES}

1. V. I. Arnol'd, Singularity theory, London Math. Soc. Lecture Note Ser., vol. 53, Cambridge Univ. Press, 1981.

2. Eivind Eriksen, Konneksjoner på en klasse moduler over simple kurvesingulariteter, Master's thesis, University of Oslo, 1994.

3. _ Differential operators on monomial curves, J. Algebra 264 (2003), no. 1, 186-198.

4. G.-M. Greuel and H. Knörrer, Einfache Kurvensingularitäten und torsionsfreie Moduln, Math. Ann. 270 (1985), 417-425.

5. A. Seidenberg, Derivations and integral closure, Pacific J. Math. 16 (1966), 167173.

6. C. T. C. Wall, Notes on the classification of singularities, Proc. London Math. Soc. (3) 48 (1984), no. 3, 461-513.

7. Yuji Yoshino, Cohen-Macaulay modules over Cohen-Macaulay rings, London Math. Soc. Lecture Note Ser., vol. 146, Cambridge Univ. Press, 1990. 